\title{
Non-additive model for specific heat of electrons
}

\author{
D.H.A.L. Anselmo ${ }^{\mathrm{a}, *}$, M.S. Vasconcelos ${ }^{\mathrm{b}}$, R. Silva $^{\mathrm{a}, \mathrm{c}}$, V.D. Mello ${ }^{\mathrm{c}}$ \\ a Universidade Federal do Rio Grande do Norte, Departamento de Física, Natal-RN, 59072-970, Brazil \\ ${ }^{\mathrm{b}}$ Escola de Ciência e Tecnologia, Universidade Federal do Rio Grande do Norte, 59072-970, Natal-RN, Brazil \\ c Universidade do Estado do Rio Grande do Norte, Departamento de Física, Mossoró-RN, 59610-210, Brazil
}

\section{A R T I C L E I N F O}

\section{Article history:}

Received 22 June 2016

Received in revised form 10 August 2016

Accepted 11 August 2016

Available online 18 August 2016

Communicated by C.R. Doering

\section{Keywords:}

Quasicrystals

Fractal spectrum

Specific heat

Non-Gaussian statistics

\begin{abstract}
A B S T R A C T
By using non-additive Tsallis entropy we demonstrate numerically that one-dimensional quasicrystals, whose energy spectra are multifractal Cantor sets, are characterized by an entropic parameter, and calculate the electronic specific heat, where we consider a non-additive entropy $S_{q}$. In our method we consider an energy spectra calculated using the one-dimensional tight binding Schrödinger equation, and their bands (or levels) are scaled onto the $[0,1]$ interval. The Tsallis' formalism is applied to the energy spectra of Fibonacci and double-period one-dimensional quasiperiodic lattices. We analytically obtain an expression for the specific heat that we consider to be more appropriate to calculate this quantity in those quasiperiodic structures.
\end{abstract}

(C) 2016 Elsevier B.V. All rights reserved.

\section{Introduction}

Since the discovery of quasicrystals by Shechtman et al. [1], awarded with the Nobel Prize, and the pioneering work of Merlin et al. [2] on the nonperiodic Fibonacci and Thue-Morse GaAs-AlAs superlattices, quasicrystals have emerged as a new form of matter. Quasicrystals are a particular type of solid that have a discrete point-group symmetry not present in Bravais lattices, like a $C_{5}$ symmetry in two dimensions, or icosahedral symmetry in three dimensions [3-6]. In one dimension (1D), the Fibonacci sequence can directly be translated into a layered quasicrystal structure, which is feasible through atomic-precision growth via molecular-beam epitaxy (MBE) [2]. Also, 1D passive photonic (and phononic) quasicrystals have been realized by MBE and other techniques. As a result of these advances, samples can now be prepared consisting of sequences of building blocks of different materials, in that the thickness and composition of individual layers can be controlled with high precision. Several interesting experimental studies have been reported in last two decades, like the transmission of bulk acoustic phonons [7], surface acoustic waves [8], photonic dispersion relation [9] and localization of light waves [10-12], to cite a few. From a theoretical point of view, the behavior of a variety of particles or quasiparticles, such as electrons [13], phonons [14, $15]$, photons [16,17], polaritons [18] and magnons [19] were inves-

\footnotetext{
* Fax: +55 8432153791.

E-mail addresses: doryh@fisica.ufrn.br (D.H.A.L. Anselmo), mvasconcelos@ect.ufrn.br (M.S. Vasconcelos), raimundosilva@fisica.ufrn.br (R. Silva), vambertodias@uern.br (V.D. Mello).
}

tigated. For example, recently, Tanese et al. [20] have reported a fractal energy spectrum of a polariton gas confined in a quasiperiodic one-dimensional cavity described by a Fibonacci sequence. On the theoretical side, very recently, one of us have reported a spinglass ordering in a two-dimensional square lattice by using the Ising model with ferromagnetic and antiferromagnetic exchange interactions following a quasiperiodic Fibonacci sequence in both directions of a square lattice [21]. A rather fascinating feature of these quasiperiodic structures is that they exhibit collective properties not shared by their constituents. Therefore, the long-range correlations induced by the construction of these systems are expected to be reflected someway in their various spectra (light propagation, electronic transmission, density of states, polaritons, etc.), defining a novel description of disorder or a new class of universality.

On the other hand, the analysis of the thermodynamic properties based on the energy spectrum derived from a fractal structure was pioneered by Tsallis and collaborators [22,23]. Their model was based on the most well-known and simple deterministic fractal geometry, the triadic Cantor set, and they showed that the specific heat of such a system exhibits a very particular behavior: it oscillates log-periodically around a mean value that equals the fractal dimension of the spectrum. Such oscillations also appear in other fractal sets $[24,25]$. Afterwards, Mauriz and collaborators $[27,28]$ have presented a model based on the polariton's and electron's multifractal energy spectra for artificial structures following the Fibonacci, Thue-Morse and double-period sequences, in order to study their thermodynamic properties. They proved that in the case of Fibonacci quasicrystals, whose incommensurate parameter 
is equal to the golden mean, there are two classes of log-periodic oscillations for the specific heat in the low temperature regime, one for the even and the other for the odd generation number of the sequence, with amplitude of the odd oscillations being bigger than the amplitude of the even one. These results are not observed in others sequences, except for a generalized Fibonacci sequence [29].

In recent years, a trend towards the non-additive statistical physics is rapidly increasing. In this context, the endeavor of the generalization of some of the conventional concepts has been under investigation. A quite interesting generalization of the conventional entropy form has been advanced by Tsallis [30] inspired by fractal and multifractal concepts. The generalized entropy possesses the usual properties of positivity, equiprobability, concavity, irreversibility and generalizes the standard additivity. This new formalism is called the nonextensive statistical mechanical formalism (NSMF) and has been proposed to treat problems with better results than the standard Boltzmann-Gibbs (BG) statistics, for instance, problems involving long-range interactions or long-range memory. The NSMF has been successfully applied to numerous concepts of statistical thermodynamics [34-39] and to many issues in the context of high energy physics [40-43], stellar polytropes [44], thermodynamic of black holes [45], relative information in cosmology [46], quantum entropies [47] and statistics of earthquakes [48].

Based on the fractal properties of these systems and also due to the long-range correlations induced by the construction of these systems, we can infer that the most appropriate statistic to study the thermodynamical properties of these complex systems is the nonextensive statistical mechanical formalism developed by Tsallis [30]. Therefore in this work we investigate the theoretical behavior of some thermodynamical quantities, namely the specific heat, free energy and entropy, calculated by considering the nonadditivity effects arising on the system.

\section{Energy spectra for quasiperiodic lattices}

In this section we briefly describe a transfer matrix treatment ${ }^{1}$ for a quasiperiodic chain which follows Fibonacci (FB) and doubleperiod (DP) rules of growth. For this purpose, we will consider a binary sequence of sites in the lattice where the potentials $V_{n}$ are arranged in a quasiperiodic fashion. By using the transfer matrix treatment, the (discrete) Schrödinger equation in the tight-binding (TB) approximation for this system can be written in the form [13]

$$
\left(\begin{array}{c}
\psi_{n+1} \\
\psi_{n}
\end{array}\right)=M(n)\left(\begin{array}{c}
\psi_{n} \\
\psi_{n-1}
\end{array}\right)
$$

where $M(n)$ is the transfer matrix of the system that makes a link from the physical properties of the $n$-th site to those of the $(n+1)$-th one. After successive applications of the transfer matrices we have $M(n)=M_{n} M_{n-1} \cdots M_{2} M_{1}$. In this way we can obtain the wave function at arbitrary sites. The numerical evaluation of the products of these transfer matrices is completely equivalent to numerically solve the Schrödinger equation for the quasiperiodic system above.

Here we consider that the potential $V_{n}$ take only two different values $V_{A}$ and $V_{B}$ arranged in accordance with the quasiperiodic Fibonacci and double-period sequences. By shifting the zero energy, we can choose, without loss of generality, these two value of the potentials to be opposite, namely $V_{A}=V$ and $V_{B}=-V$, where $V$ is the potential strength.

The Fibonacci structure can be grown experimentally by juxtaposition of layers $\mathrm{A}$ and $\mathrm{B}$, such as this superlattice can be

\footnotetext{
1 See ref. [49] for an example of transfer matrices applied to Cantor sets.
}

constructed recursively as follow: $S_{n+1}=S_{n} S_{n-1}$ for $n \geq 1$, with $S_{0}=B$ and $S_{1}=A$. This recursion rule is equivalent to the inflation rule $A \rightarrow A B$ and $B \rightarrow A$. This sequence increases with the Fibonacci number $F_{n}$, defined by $F_{n}=F_{n-1}+F_{n-2}$, with the initial conditions $F_{0}=F_{1}=1$. The ratio $F_{n} / F_{n-1}$ for increasing $n$ converges towards the golden mean $\tau=(1 / 2)(1+\sqrt{5})$. Analogously, the $n$-th generation of the double-period sequence can be obtained from the relations $S_{n}=S_{n-1} S_{n-1}^{\dagger}$, and $S_{n}^{\dagger}=S_{n-1} S_{n-1}($ for $\mathrm{n} \geq 1$ ). The number of letters in this sequence increases as $2^{n}$, and the initial conditions are $S_{0}=A$ and $S_{0}^{\dagger}=B$. Its inflation rule is given by the transformations $A \rightarrow A B, B \rightarrow A A$.

For the FB quasiperiodic lattice, the transfer matrix is given by the product of $F_{n}$ matrices $M_{j}(j=A, B)$, given by

$M_{j}=\left(\begin{array}{cc}E-V_{j} & -1 \\ 1 & 0\end{array}\right)$.

It is easily shown that $M_{n}$ obeys [13]

$M_{n+1}=M_{n-1} M_{n}$

with $M_{0}=M_{B}$ and $M_{1}=M_{A}$. The double-period (DP) sequence starting matrices are analogous to the Fibonacci sequence, i.e., in our notation, $S_{0}=M_{1}=M_{A}$ and $S_{0}^{\dagger}=M_{0}=M_{B}$. Note that Eq. (3) depends on energy $E$. Since the determinant of $M_{n}$ is the unit, the energy spectrum of the system is determined by $E$ values which satisfy $\left|\operatorname{Tr}\left(M_{n}\right)\right| \leq 2$, where $\operatorname{Tr}()$ means the trace of a matrix. This is equivalent to look for energies where the solutions $\psi_{n}$ do not grow exponentially. Once obtained, this energy spectra allow us to calculate the specific heat given by the system's allowed bands.

The first connection with physical properties of quasicrystalline superlattices was made by Kohmoto and Banavar [13]. In their work the authors obtain a set of recursion relations and selfconsistent maps. These equations define the trace map of the system and are the key for the study of the wavefunction localization and spectral properties. With the aid of those relations and the initial conditions one can determinate the real allowed values of the energy for a given generation number $n$. Numerical examples for FB and DP quasiperiodic spectra are shown in the references [27, 28]. In the next section we will apply these rules to numerically obtain the specific heat.

\section{Specific heat}

It is our intention in this section to discuss in detail how to obtain an expression for the generalized entropy and specific heat, from the energy spectrum. The starting point for this is the energy spectrum for the continuous fractal set, depicted in Fig. 1, for the FB and DP sequences, where

$\Delta_{i}=\epsilon_{2 i}-\epsilon_{2 i-1} \Longrightarrow \epsilon_{2 i}=\epsilon_{2 i-1}+\Delta_{i}$.

Thus, when we scale the spectra above onto the $[0,1]$ interval, we can see that $n=1$ (where $n$ is the generation number) corresponds to a continuous spectrum going from $\epsilon_{1}$ to $\left(\epsilon_{1}+\Delta_{1}\right) ; n=2$ corresponds to a spectrum whose first branch goes from $\epsilon_{1}$ to $\epsilon_{2}=\left(\epsilon_{1}+\Delta_{1}\right)$ and the second one goes from $\epsilon_{3}$ to $\epsilon_{4}=\left(\epsilon_{3}+\Delta_{2}\right)$ and so on for increasing $n$. We take the level density (DOS) inside each band to be constant, and the same for all bands in a given hierarchy. In this case, a fractal emerges at the $n \rightarrow \infty$ limit. One can, however, make a detailed study considering the influence of a non-uniform DOS [26]. For examples of energy spectra of other quasiperiodic sequences see, e.g., [27-29].

To study such complex systems, we use a non-additive entropy which was introduced by Tsallis in 1988 [30] and was later 

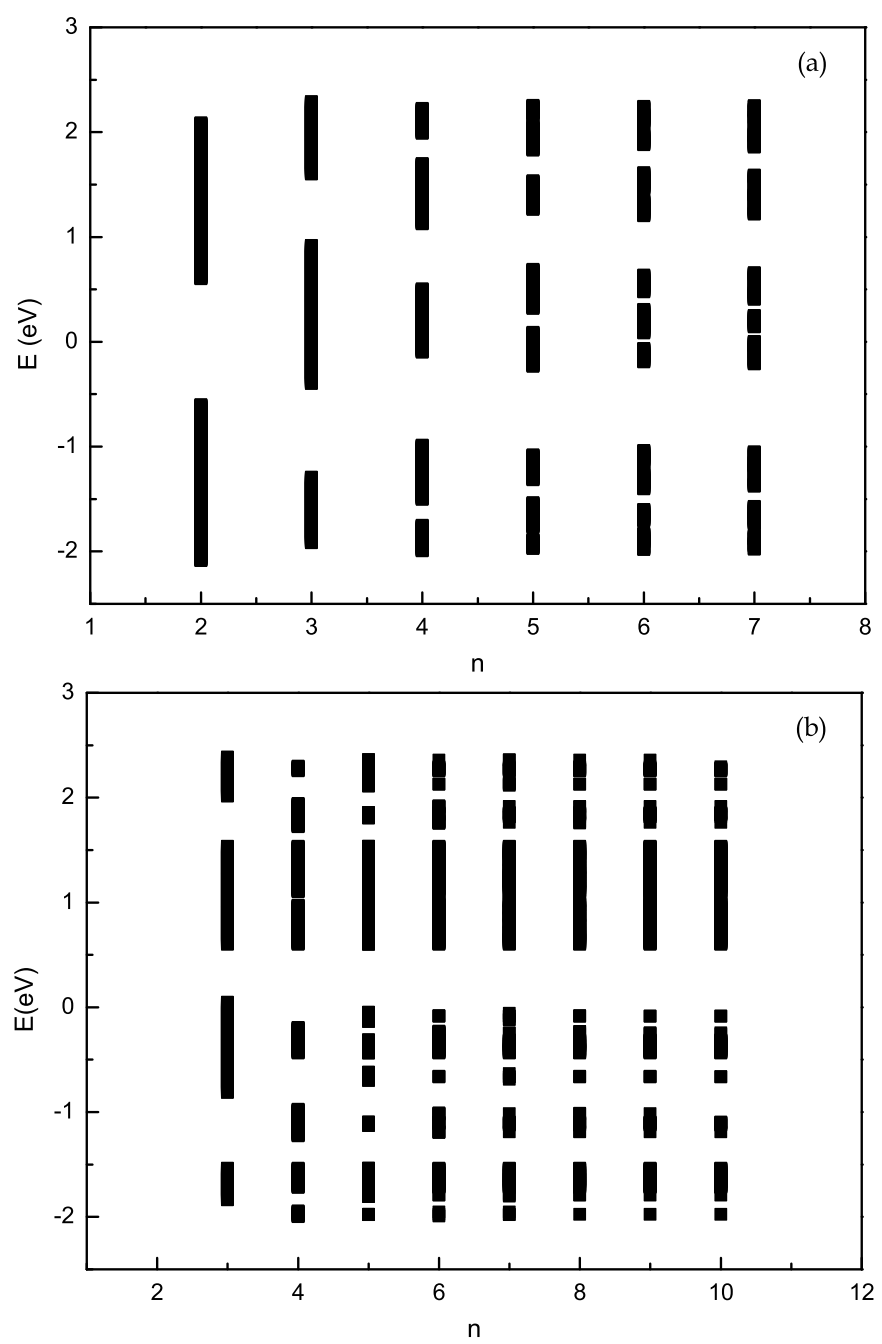

Fig. 1. Energy spectra. (a) Solutions to the TB equation for the FB sequence. (b) Same for the DP sequence.

adopted and refined in diverse fields demonstrating power law dynamics and fractality [31]. The non-additive entropy is therefore defined as

$$
\begin{aligned}
S_{q} & =k_{B} \frac{1-\sum_{i=1}^{W} p_{i}^{q}}{(q-1)} \quad \text { for } q \neq 1 \\
& =-\sum_{i=1}^{W} p_{i} \ln p_{i} \quad \text { for } q=1
\end{aligned}
$$

where $p_{i}$ denotes the probabilities of the $i$-th microscopic states, the average runs over the total number of states $W$ and $q$ is the exponent (also known as entropic parameter) which characterizes the particular statistics. Note that for $q=1$ the classical BoltzmannGibbs (BG) statistics is recovered and thus a departure of the exponent $q$ from the value 1 signals a departure from BG statistics.

In the canonical ensemble of the generalized statistic, the energy can fluctuate around a value $\langle E\rangle_{q}$, defined by $[52,53]$

$U_{q} \equiv\langle E\rangle_{q}=\sum_{i=1}^{W} p_{i}^{q} E_{i}$.

Here, let us emphasize that there are at least three generalizations of Boltzmann-Gibbs statistics based on calculation of the mean energy in the context of canonical ensemble, or analogous observables for grand-canonical ensembles [54]. In Ref. [55], on the other hand, the authors have shown an equivalence among all versions of Tsallis framework. More recently, the use of the escortaveraging scheme, which consider the definition $U_{q} \equiv\langle E\rangle_{q}=$ $\frac{\sum_{i=1}^{W} p_{i}^{q} E_{i}}{\sum_{i=1}^{W} p_{i}^{q}}$, has been disproved, i.e., the escort distribution seems to be unnecessary in the context of Tsallis statistics [56]. Therefore, our approach shall follow the generalization given by Eq. (6).

This definition generates the probability distribution given by

$p_{i}=\frac{1}{Z_{q}}\left[1-(1-q) \frac{E_{i}}{k_{B} T}\right]^{\frac{1}{1-q}} \equiv \frac{1}{Z_{q}} e^{-\frac{E_{i}}{k_{B} T}}$

where $Z_{q}$ is the generalized partition function which ensures the normalization of the probability. Note that the generalization given by Eq. (6) provides the same results obtained through constraint $U_{q} \equiv\langle E\rangle_{q}=\sum_{i=1}^{W} p_{i} E_{i}$, by using the transformation $q \rightarrow(2-q)$. In Ref. [56], the authors demonstrated that this constraint, together with the ordinary normalization $\sum_{i=1}^{W} p_{i}=1$, describes the complete Tsallis framework, with $S_{q \in(0,1]}$ and $S_{q \in[1,2)}$. By considering the generalized partition function for a single energy band, starting in $\epsilon_{1}$ and finishing in $\epsilon_{2}$, we can calculate it by

$Z_{q}=\int_{\epsilon_{1}}^{\epsilon_{2}} \rho(\epsilon)[1-\beta(1-q) \epsilon]^{\frac{1}{1-q}} d \epsilon$

where $\beta=1 / T$. We are also considering Boltzmann's constant $k_{B}=1$, and we take the density of states $\rho(\epsilon)=1$. The symbol $e_{q}$ stands for the generalized $q$-exponential, which is defined by

$e_{q}(x) \equiv[1+(1-q) x]^{\frac{1}{1-q}}$.

In particular, $e_{q}^{x}=e^{x}$ when $q$ goes to 1 . Here emerges a fundamental difference between the NSMF and the BG formalism. In the later, the distribution of probability is given by an exponential, while in the former, the distribution of probability obeys a power law. If $q>1$, the distribution have a smoother decaying than the exponential function (as a negative argument, as in the case of the BG formalism). This makes the states with higher power to be visited more frequently in the NSMF than in BG statistics. If $q<1$, the situation is reversed and the equation (7) decay is much more pronounced than an exponential, presenting negative (or imaginary) values of the probability, when

$\frac{E_{i}}{k_{B} T}>\frac{1}{1-q}$,

which is physically unacceptable. Some particular values of $q<1$ present probabilities increasing with energy, when (10) is satisfied, which is also unacceptable physically. To fix this problem, Plastino et al. introduced a cut-off [53] distribution of probabilities, which requires $P_{i}=0$ when the condition in equation (10) is satisfied. In this case, for $q<1$, the distribution of probabilities exhibits a thermal cutoff on the maximum value allowed for the energy, which is given by $E_{\max }=k_{B} T /(1-q)$.

Let us now make the connection with the thermodynamical quantities. The definition of the entropy $S_{q}$, by using the same processes used in the calculus of the extremes of the BG entropy in the canonical (or grand-canonical) ensembles, allow us to obtain the link between the non-extensive entropy and a set of thermodynamical quantities, as temperature and potentials.

In this regard, considering the energy given by Eq. (6), and taking $T=1 /\left(k_{B} \beta\right)$ it is easy to show that

$\frac{1}{T}=\frac{\partial S_{q}}{\partial U_{q}}$

$F_{q}=U_{q}-T S_{q}=-\frac{1}{\beta} \ln _{q} Z_{q}$, 
$U_{q}=-\frac{\partial\left[\ln _{q} Z_{q}\right]}{\partial \beta}$

and

$C_{q}=\frac{\partial U_{q}}{\partial T}$.

Now we can calculate the thermodynamic quantities from the equations above and from the partition function. By solving the integral in equation (8) (with $\rho(\varepsilon)=1$ and $k_{B}=1$, for simplicity), it is easy to show that the partition function for the fractal (banded) energy spectra depicted in Fig. 1 is given by

$Z_{q}=\frac{1}{\beta(2-q)} \sum_{i=1,3,5 \ldots}^{2 N-1}\left[e_{q}^{2-q}\left(-\beta \varepsilon_{i}\right)-e_{q}^{2-q}\left(-\beta \varepsilon_{i+1}\right)\right]$,

where $q<2$ and $e_{q}^{2-q}\left(-\beta \varepsilon_{i}\right)>e_{q}^{2-q}\left(-\beta \varepsilon_{i+1}\right)$ to ensure that $Z_{q}>0$. $N$ is the number of bands in the fractal spectrum. Following the works developed by Borges [32], and Nivanen et al. [33] instead of using the natural logarithm, we make use of the generalized logarithm, as follows

$C_{q}(T)=\beta^{2} \frac{\partial^{2} \ln _{q} Z_{q}}{\partial \beta^{2}}$

In the above expressions, $\ln _{q}$ is the $q$-logarithm, given by

$\ln _{q}(x)=\frac{x^{1-q}-1}{1-q}$

It is our intent here to study the specific heat of this system. An analogue study have been reported by Moreira et al. [50], but in their work the authors did not use the generalized $q$-logarithm, which should be the adequate mathematical formalism [52]. Therefore, using the generalized equation for the partition function $Z_{q}$, defined in Eq. (15), and the equation for the generalized specific heat in Eq. (16), we can find the first slope of $\ln _{q} Z_{q}$, that is given by

$\frac{\partial \ln _{q} Z_{q}}{\partial T}=Z^{-q} \frac{\partial Z_{q}}{\partial T}$.

By organizing in terms of powers of $Z_{q}$, we obtain the expression for the generalized specific heat as follows

$C_{q}(T)=(2-q) Z_{q}^{1-q}+\frac{2(1-q) g_{q N}+\beta f_{q N}}{Z_{q}}-q \frac{g_{q N}^{2}}{Z_{q}^{1+q}}$,

where $g_{q N}$ and $f_{q N}$ are functions of the entropic parameter $q$, and are given by

$$
\begin{aligned}
g_{q N} & =\sum_{i=1,3,5 \ldots}^{2 N-1}\left[-\varepsilon_{i} e_{q}\left(-\beta \varepsilon_{i}\right)+\varepsilon_{i+1} e_{q}\left(-\beta \varepsilon_{i+1}\right)\right], \\
f_{q N} & =\sum_{i=1,3,5 \ldots}^{2 N-1}\left[\varepsilon_{i}^{2} e_{q}^{q}\left(-\beta \varepsilon_{i}\right)-\varepsilon_{i+1}^{2} e_{q}^{q}\left(-\beta \varepsilon_{i+1}\right)\right] .
\end{aligned}
$$

Note that the expression to the generalized specific heat reduces to the extensive specific heat obtained in previous works [27-29], when $q \rightarrow 1$. Also the expressions to $g_{q N}$ and $f_{q N}$ reduces to the standard expressions in the works of Mauriz et al. [27-29] and Moreira et al. [50].

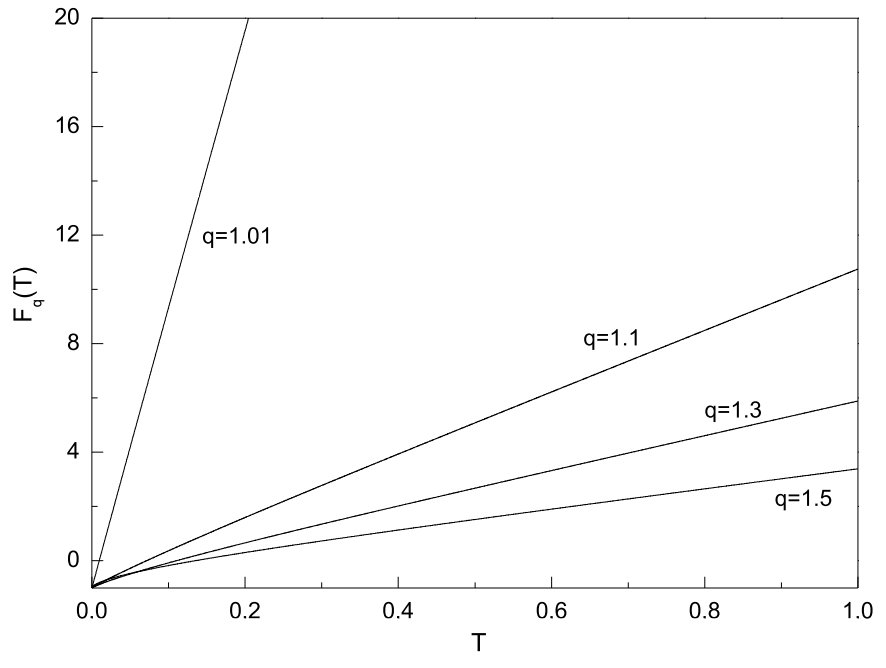

Fig. 2. Gibbs free energy as function of the temperature for the 13th Fibonacci generation.

\section{Numerical results}

We now address the entropy, the specific heat, and the Gibbs free energy obtained from the theoretical model described in the previous section. For the calculated energy spectra we have used the tight binding Schrödinger equation together with the transfermatrix approach and the physical parameters used in Ref. [27]. From those banded spectra we have used Eqs. (12) to (21) to obtain all the results below. In Fig. 2 we show the Gibbs free energy, as a function of the temperature (in units of $k_{B}$ ). In this figure we can observe the linear behavior for values of the $q$ exponent close to the unity, as expected. For example, the temperature dependence of the Gibbs free energy for an ideal gas is given by the Gibbs-Helmholtz equation, which is linear in the temperature. For values of $q>1$, the Gibbs free energy has a non-linear behavior.

The new outcome here is the clear influence of the strength of the $q$ parameter, when we analyze both specific heat and entropy. When the specific heat calculated as function of temperature is analyzed, for odd and even generations of the FB sequence (the behavior for the PD sequence is fairly analogous), it shows a fairly strong resemblance with the BG standard statistics results when the entropic parameter $q$ is still close to unity, and one can notice the existence of two classes of oscillations, for odd and even generation numbers of FB sequence. The pattern of the odd generation oscillations have greater amplitudes than the even ones. Also, that the oscillations occurs around an average value, and this can be interpreted as a superposition of Schottky anomalies, corresponding to different scales of the energy spectrum. In Fig. 3a several curves are shown, now for a fixed generation of the FB sequence. As the $q$ value is increased, the oscillation pattern disappears, which indicates that for higher $q$ values, the higher energy terms contribute strongly to the specific heat, a result that is not usually expected. So there should be, at least in principle, a value of $q$ that is more appropriate to describe the distribution of energies among the several states. In Fig. $3 \mathrm{~b}$ we show the $\log -\log$ plot of specific heat versus temperature for several generations of a quasiperiodic unidimensional double-period superlattice, for a constant value of the entropic parameter $q=1.2$. The parity pattern of oscillations in the specific heat, present in the FB sequence, was not observed in the DP sequence. The size of the unit-cell in the DP superlattice grows as $2^{N}$, where $N$ is the generation number. Although this is different from the FB sequence, we do not think that this is the cause to such absence of parity oscillations. Here, as in the FB case, the higher the value of $q$, the higher the damping in the oscillations. In any case, however, the specific heat decreases as $T^{-2}$, indepen- 

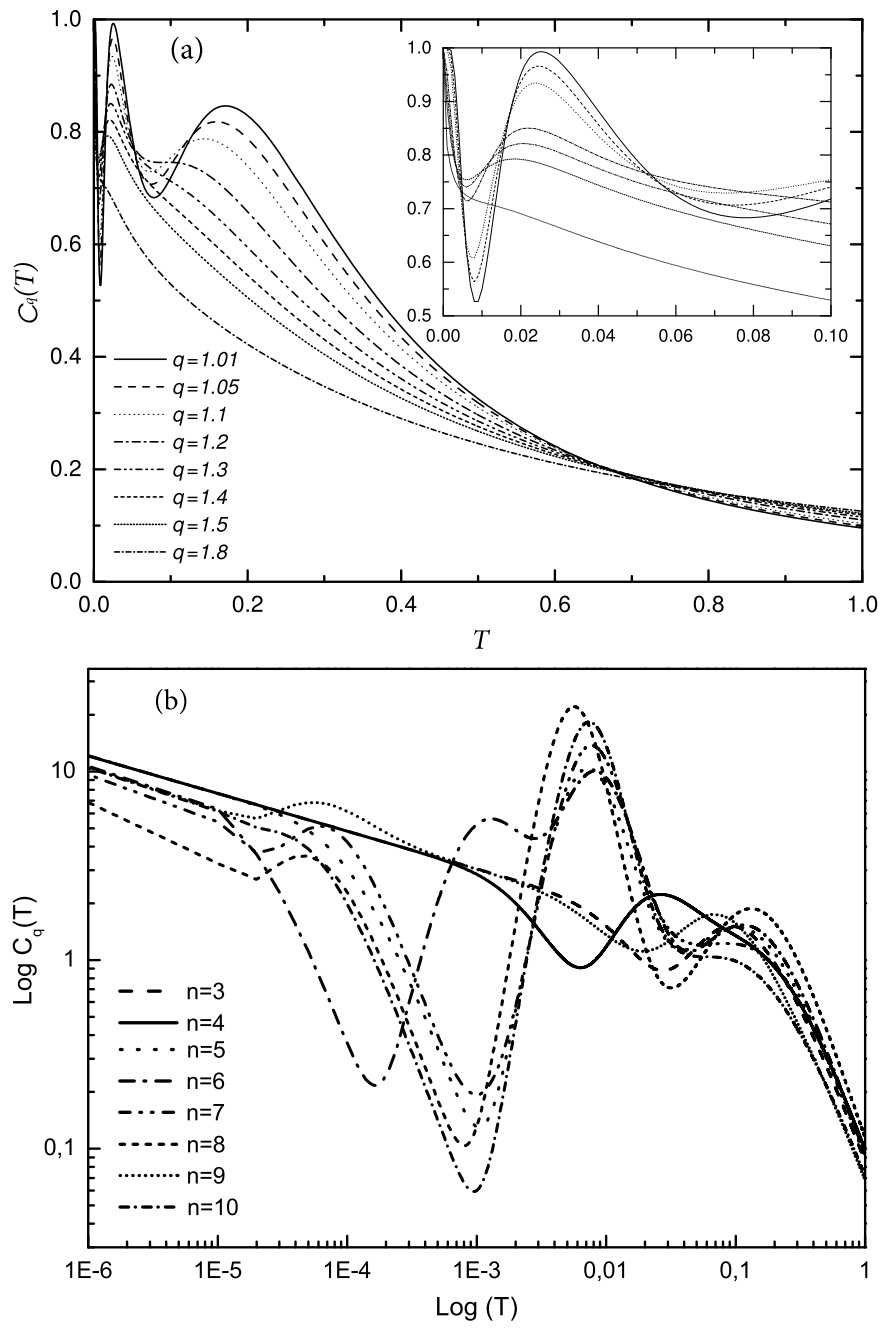

Fig. 3. (a) Specific heat as function of the temperature for the 5th Fibonacci generation, for several values of $q \in[1,2]$. The inset details the oscillations pattern. (b) Log-log plot of specific heat versus temperature for several generations of a quasiperiodic unidimensional double-period superlattice, for $q=1.2$.

dently of the value of the nonextensive parameter $q$. In the work of Provata [51] the author argue that, in the context of fractal sets (which is just the behavior of our energy spectra) an appropriate $q$ is the one for which the corresponding entropy increases linearly with the system size L. Therefore, in order to give some indication of the (non)extensivity of this quasiperiodic system, we have plotted in Fig. 4 the logarithm of the generalized entropy $S_{q}$ as function of the logarithm of the size of the system, which for the Fibonacci superlattice is given by the Fibonacci number $F_{n}=F_{n-1}+$ $F_{n-2}$, for two values of the temperature. A purely linear behavior would give a straight line (in this scale) with unitary angular coefficient $\alpha$. As one can see, all the curves have higher values of $\alpha$, therefore there is no value of $q$, in the physical region $q \in[1,2]$, for which the systems behave extensively. Analogous results were obtained for the DP sequence, not shown here. Also, for higher temperatures, the specific heat decreases as $T^{-2}$, independently of the value of the nonextensive parameter, which is usually a typical characteristic of systems with a bounded energy spectrum.

\section{Conclusions}

The non-additive entropy have been used to show numerically the introduction of effects of strong statistical correlations and fractal behavior on one-dimensional quasicrystals. Such analysis have shown that the measure of non-additivity, characterized by
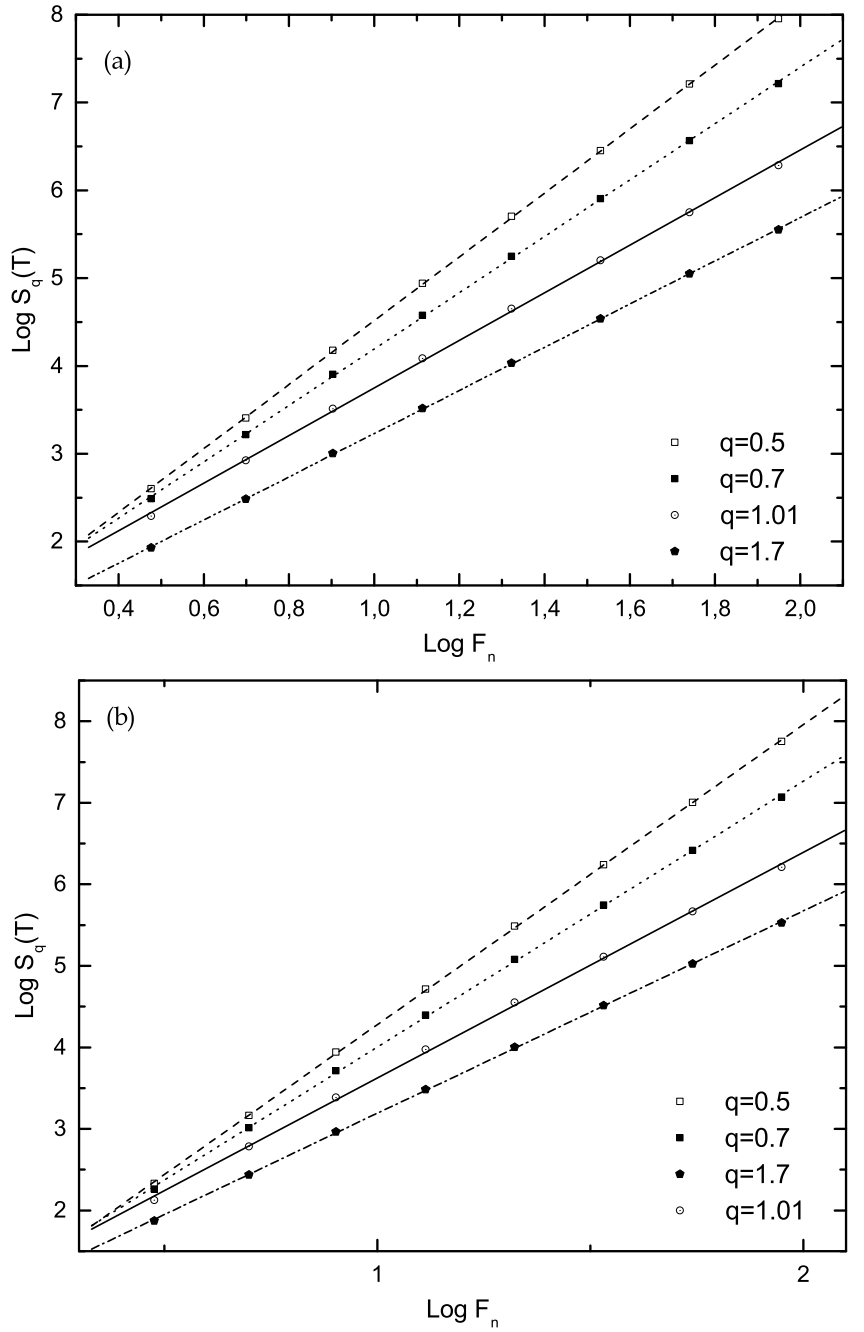

Fig. 4. Log-log plot of the generalized entropy $S_{q}$ as a function of the Fibonacci generation number, for two values of temperature: (a) $T=0.5 \mathrm{~K}$, (b) $T=1.8 \mathrm{~K}$.

a q-entropy, plays an important role on the thermodynamic properties of this system.

Some thermodynamical quantities, namely, the free Gibbs energy, the entropy and the specific heat, were obtained from the solutions of the tight-biding equation for the electronic energy spectra of quasiperiodic Fibonacci and double-period unidimensional superlattices. Our main results are the equation (19), which generalizes several previous results (where the authors did not make use of the appropriate nonextensive algebra) and the absence of a $q$ value which makes the system extensive, as reported in other works $[51,57]$. At higher temperatures, the specific heat decreases as $T^{-2}$, which is a typical characteristic of systems with a bounded energy spectrum. The increase of the entropic parameter $q$ leads to a damping in the oscillatory behavior, reinforcing the absence of extensive behavior of the entropy $S_{q}$. Also, the parity pattern of oscillations in the specific heat, present in the FB sequence, was not observed in the PD sequence.

It is worth mentioning that from the non-additivity thermodynamical viewpoint, Gibbs free energy and Specific heat are consistent with the interval obtained through second and third laws of thermodynamics, i.e. the entropic parameter belonging to interval $0<q<2[34,38,56,58]$.

Finally, we intend to consider our approach to investigate the low-temperature specific heat spectra of long-range correlated quasiperiodic DNA molecules. This issue will be addressed in a forthcoming communication. 


\section{Acknowledgements}

\section{We would like to thank the Brazilian Research Agencies CNPq and FINEP for partial financial support.}

\section{References}

[1] D. Shechtman, I. Blech, D. Gratias, J.W. Cahn, Metallic phase with long-range orientational order and no translational symmetry, Phys. Rev. Lett. 53 (1984) 1951-1953.

[2] R. Merlin, K. Bajema, R. Clarke, F.Y. Juang, P.K. Bhattacharya, Quasiperiodic GaAs-AlAs heterostructures, Phys. Rev. Lett. 55 (1985) 1768-1770.

[3] H.C. Jeong, P.J. Steinhardt, Finite-temperature elasticity phase transition in decagonal quasicrystals, Phys. Rev. B 48 (1993) 9394-9403.

[4] D. Levine, P.J. Steinhardt, Quasicrystals: a new class of ordered structures, Phys. Rev. Lett. 53 (1984) 2477-2480.

[5] P. Monceau, J.C.S. Levy, M. Perreau, Statistics of local environments in fractals, Phys. Lett. A 171 (1992) 167-171.

[6] C. Godrèche, J.M. Luck, Quasiperiodicity and randomness in tilings of the plane, J. Stat. Phys. 55 (1/2) (1988).

[7] D.C. Hurley, S. Tamura, J.P. Wolfe, K. Ploog, J. Nagle, Angular dependence of phonon transmission through a Fibonacci superlattice, Phys. Rev. B 37 (1988) 8829-8836.

[8] L. Maeon, J.P. Desieleri, D. Sornette, Localization of surface acoustic waves in a one-dimensional quasicrystal, Phys. Rev. B 44 (1991) 6755-6772.

[9] T. Hattori, N. Tsurumachi, S. Kawato, H. Nakatsuka, Photonic dispersion relation in a one-dimensional quasicrystal, Phys. Rev. B 50 (1994) 4220-4223.

[10] R.W. Peng, X.Q. Huang, F. Qiu, Wang Mu, A. Hu, S.S. Jiang, M. Mazzera, Symmetry-induced perfect transmission of light waves in quasiperiodic dielectric multilayers, Appl. Phys. Lett. 80 (2002) 3063-3065.

[11] L.D. Negro, C.J. Oton, Z. Gaburro, L. Pavesi, P. Johnson, A. Lagendijk, R. Righini, M. Colocci, D.S. Wiersma, Light transport through the band-edge states of Fibonacci quasicrystals, Phys. Rev. Lett. 90 (2003) 055501.

[12] W. Gellermann, M. Kohmoto, B. Sutherland, P.C. Taylor, Localization of light waves in Fibonacci dielectric multilayers, Phys. Rev. Lett. 72 (1994) 633-636.

[13] M. Kohmoto, J.R. Banavar, Quasiperiodic lattice: electronic properties, phonon properties, and diffusion, Phys. Rev. B 34 (1986) 563-566;

M. Kohmoto, B. Sutherland, K. Iguchi, Localization of optics: quasiperiodic media, Phys. Rev. Lett. 58 (1987) 2436-2438;

M. Kohmoto, B. Sutherland, C. Tang, Critical wave functions and a Cantor-set spectrum of a one-dimensional quasicrystal model, Phys. Rev. B 35 (1987) 1020-1033.

[14] M. Quilichini, Phonon excitations in quasicrystals, Rev. Mod. Phys. 69 (1997) 277-314.

[15] M. Kolár, M.K. Ali, Attractors of some volume-nonpreserving Fibonacci trace maps, Phys. Rev. A 39 (1989) 6538-6544;

M. Kolár, M.K. Ali, One-dimensional generalized Fibonacci tilings, Phys. Rev. B 41 (1990) 7108-7112;

M. Kolár, M.K. Ali, F. Nori, Generalized Thue-Morse chains and their physical properties, Phys. Rev. B 43 (1991) 1034-1047.

[16] M.S. Vasconcelos, E.L. Albuquerque, A.M. Mariz, Optical localization in quasiperiodic multilayers, J. Phys. Condens. Matter 10 (1998) 5839.

[17] E.R. Brandão, C.H. Costa, M.S. Vasconcelos, D.H.A.L. Anselmo, V.D. Mello, Octonacci photonic quasicrystals, Opt. Mater. 46 (2016) 378-383.

[18] M.S. Vasconcelos, E.L. Albuquerque, Plasmon-polariton fractal spectra in quasiperiodic multilayers, Phys. Rev. B 57 (1998) 2826-2833.

[19] I.P. Coelho, M.S. Vasconcelos, C.G. Bezerra, Transmission fingerprints in quasiperiodic magnonic multilayers, J. Magn. Magn. Mater. 323 (2011) 3162-3167.

[20] D. Tanese, E. Gurevich, F. Baboux, T. Jacqmin, A. Lemaître, E. Galopin, I. Sagnes, A. Amo, J. Bloch, E. Akkermans, Fractal energy spectrum of a polariton gas in a Fibonacci quasiperiodic potential, Phys. Rev. Lett. 112 (2014) 146404.

[21] G.A. Alves, M.S. Vasconcelos, T.F.A. Alves, Critical properties of a twodimensional Ising magnet with quasiperiodic interactions, Phys. Rev. E 93 (2016) 042111.

[22] C. Tsallis, L.R. da Silva, R.S. Mendes, R.O. Vallejos, A.M. Mariz, Specific heat anomalies associated with Cantor-set energy spectra, Phys. Rev. E 56 (1997) R4922-R4925.

[23] R.O. Vallejos, R.S. Mendes, L.R. da Silva, C. Tsallis, Connection between energy spectrum, self-similarity, and specific heat log-periodicity, Phys. Rev. E 58 (1998) 1346-1351.

[24] D. Sornette, Discrete-scale invariance and complex dimensions, Phys. Rep. 297 (1998) 239-270

[25] E. Akkermans, G.V. Dunne, A. Teplyaev, Physical consequences of complex dimensions of fractals, Europhys. Lett. 88 (2009) 40007.

[26] P. Monceau, J.C.S. Levy, Effects of deterministic and random discrete scale invariance on spin wave spectra, Physica E 44 (2012) 1697-1702.
[27] P.W. Mauriz, E.L. Albuquerque, M.S. Vasconcelos, Specific heat properties of polariton modes in quasicrystals, Phys. Rev. B 63 (2001) 184203.

[28] P.W. Mauriz, E.L. Albuquerque, M.S. Vasconcelos, Electronic specific heat properties in one-dimensional quasicrystals, Physica A 294 (2001) 403-414.

[29] P.W. Mauriz, M.S. Vasconcelos, E.L. Albuquerque, Specific heat properties of electrons in generalized Fibonacci quasicrystals, Physica A 329 (2003) 101-113.

[30] C. Tsallis, Possible generalization of Boltzmann-Gibbs statistics, J. Stat. Phys. 52 (1988) 479-487.

[31] M. Gell-Mann, C. Tsallis (Eds.), Nonextensive Entropy: Interdisciplinary Applications, Oxford University Press, New York, 2004.

[32] E.P. Borges, A possible deformed algebra and calculus inspired in nonextensive thermostatistics, Physica A 340 (2004) 95-101.

[33] L. Nivanen, A. Le Méhauté, Q.A. Wang, Generalized algebra within a nonextensive statistics, Rep. Math. Phys. 52 (2003) 437-444.

[34] R. Silva, D.H.A.L. Anselmo, J.S. Alcaniz, Nonextensive quantum H-theorem, Europhys. Lett. 89 (2010) 10004

[35] M.S. Ribeiro, C. Tsallis, F.D. Nobre, Probability distributions extremizing the nonadditive entropy $S_{\delta}$ and stationary states of the corresponding nonlinear Fokker-Planck equation, Phys. Rev. E 88 (2013) 052107.

[36] L.J.L. Cirto, L.S. Lima, F.D. Nobre, Controlling the range of interactions in the classical inertial ferromagnetic Heisenberg model: analysis of metastable states, J. Stat. Mech. 2015 (2015) P04012.

[37] J.S. Andrade Jr., G.F.T. da Silva, A.A. Moreira, F.D. Nobre, E.M.F. Curado, Thermostatistics of overdamped motion of interacting particles, Phys. Rev. Lett. 105 (2010) 260601.

[38] E.P. Bento, G.M. Viswanathan, M.G.E. da Luz, R. Silva, Third law of thermodynamics as a key test of generalized entropies, Phys. Rev. E 91 (2015) 022105.

[39] R. Hanel, S. Thurner, M. Gell-Mann, How multiplicity determines entropy and the derivation of the maximum entropy principle for complex systems, Proc. Natl. Acad. Sci. USA 111 (19) (2014) 6905-6910.

[40] A.A. Bylinkin, N.S. Chernyavskaya, A.A. Rostovtsev, Predictions on the transverse momentum spectra for charged particle production at LHC-energies from a two component model, Eur. Phys. J. C 75 (2015) 166.

[41] F.H. Liu, Y.Q. Gao, B.C. Li, Comparing two-Boltzmann distribution and Tsallis statistics of particle transverse momentums in collisions at LHC energies, Eur. Phys. J. A 50 (2014) 123.

[42] H.R. Wei, F.H. Liu, R.A. Lacey, Kinetic freeze-out temperature and flow velocity extracted from transverse momentum spectra of final-state light flavor particles produced in collisions at RHIC and LHC, Eur. Phys. J. A 52 (2016) 102.

[43] M. Praszalowicz, A. Francuz, Geometrical scaling in inelastic inclusive particle production at the LHC, Phys. Rev. D 92 (2015) 074036.

[44] G. Livadiotis, Superposition of polytropes in the inner heliosheath, Astrophys. J. Suppl. Ser. 223 (2016) 13.

[45] V.G. Czinner, H. Iguchi, Rényi entropy and the thermodynamic stability of black holes, Phys. Lett. B 752 (2016) 306.

[46] V.G. Czinner, F.C. Mena, Relative information entropy in cosmology: the problem of information entanglement, Phys. Lett. B 758 (2016) 9.

[47] J.A. Carrasco, F. Finkel, A. Gonzalez-Lopez, M.A. Rodriguez, P. Tempesta, Generalized isotropic Lipkin-Meshkov-Glick models: ground state entanglement and quantum entropies, J. Stat. Mech. 2016 (2016) 033114.

[48] T.M. Scherrer, G.S. Franca, R. Silva, D.B. de Freitas, C.S. Vilar, Analysis of four Brazilian seismic areas using a nonextensive approach, Europhys. Lett. 109 (2015) 49001

[49] M. Perreau, J.C.S. Levy, Randomness in fractals, connectivity dimensions, and percolation, Phys. Rev. A 40 (1989) 4690.

[50] D.A. Moreira, E.L. Albuquerque, L.R. da Silva, D.S. Galvão, Low-temperature specific heat spectra considering nonextensive long-range correlated quasiperiodic DNA molecules, Physica A 387 (2008) 5477-5482.

[51] A. Provata, Non-extensive block entropy statistics of Cantor fractal sets, Physica A 381 (2007) 148-154.

[52] E.M.F. Curado, C. Tsallis, Generalized statistical mechanics: connection with thermodynamics, J. Phys. A, Math. Gen. 25 (1991) 1019.

[53] A.R. Plastino, A. Plastino, From Gibbs microcanonical ensemble to Tsallis generalized canonical distribution, Phys. Lett. A 193 (1994) 140-143.

[54] C. Tsallis, R.S. Mendes, A.R. Plastino, The role of constraints within generalized nonextensive statistics, Physica A 261 (1998) 534-554.

[55] G.L. Ferri, D. Martinez, A. Plastino, Equivalence of the four versions of Tsallis's statistics, J. Stat. Mech. 2005 (2005) P04009.

[56] G.B. Bagci, T. Oikonomou, Validity of the third law of thermodynamics for the Tsallis entropy, Phys. Rev. E 93 (2016) 022112.

[57] N.T.C.M. Souza, D.H.A.L. Anselmo, V.D. Mello, R. Silva, Analysis of fractal groups of the type d-(m,r)-Cantor within the framework of Kaniadakis statistics, Phys. Lett. A 378 (2014) 1691-1694.

[58] S. Abe, A.K. Rajagopal, Validity of the second law in nonextensive quantum thermodynamics, Phys. Rev. Lett. 91 (2003) 120601. 